\title{
Powerful Molecular Outflows in Nearby Active Galaxies
}

\author{
Sylvain Veilleux ${ }^{1}$, Marcio Meléndez ${ }^{1}$, and the SHINING Team \\ ${ }^{1}$ Department of Astronomy, University of Maryland, College Park, MD 20742, USA \\ email: veilleux@astro.umd.edu
}

\begin{abstract}
We report the results from a systematic search for molecular (OH $119 \mu \mathrm{m})$ outflows with Herschel-PACS $\dagger$ in a sample of 43 nearby $(z<0.3)$ galaxy mergers, mostly ultraluminous infrared galaxies (ULIRGs) and QSOs. We find that the character of the $\mathrm{OH}$ feature (strength of the absorption relative to the emission) correlates with that of the 9.7- $\mu \mathrm{m}$ silicate feature, a measure of obscuration in ULIRGs. Unambiguous evidence for molecular outflows, based on the detection of $\mathrm{OH}$ absorption profiles with median velocities more blueshifted than $-50 \mathrm{~km}$ $\mathrm{s}^{-1}$, is seen in $26(70 \%)$ of the $37 \mathrm{OH}$-detected targets, suggesting a wide-angle $\left(\sim 145^{\circ}\right)$ outflow geometry. Conversely, unambiguous evidence for molecular inflows, based on the detection of $\mathrm{OH}$ absorption profiles with median velocities more redshifted than $+50 \mathrm{~km} \mathrm{~s}^{-1}$, is seen in only 4 objects, suggesting a planar or filamentary geometry for the inflowing gas. Terminal outflow velocities of $\sim-1000 \mathrm{~km} \mathrm{~s}^{-1}$ are measured in several objects, but median outflow velocities are typically $\sim-200 \mathrm{~km} \mathrm{~s}^{-1}$. While the outflow velocities show no statistically significant dependence on the star formation rate, they are distinctly more blueshifted among systems with large AGN fractions and luminosities $\left[\log \left(L_{\mathrm{AGN}} / L_{\odot}\right) \geqslant 11.8 \pm 0.3\right]$. The quasars in these systems play a dominant role in driving the molecular outflows. In contrast, the most AGN dominated systems, where $\mathrm{OH}$ is seen purely in emission, show relatively modest $\mathrm{OH}$ line widths, despite their large AGN luminosities, perhaps indicating that molecular outflows subside once the quasar has cleared a path through the obscuring material.
\end{abstract}

Keywords. galaxies: active, galaxies: evolution, ISM: jets and outflows, ISM: molecules, quasars: general

\section{Introduction}

Studies conducted in the past three years are shedding new light on the wind phenomenon in the local universe: (1) Early results from our Herschel guaranteed time key program SHINING (PI Sturm) have revealed far-infrared (FIR) OH features with PCygni profiles indicative of massive molecular outflows in a number of ULIRGs (Fischer et al. 2010; Sturm et al. 2011, hereafter F10 and S11, respectively; also González-Alfonso et al. 2012; González-Alfonso et al. 2014). In a few of these objects, particularly those with dominant AGN such as Mrk 231, the nearest quasar known, the terminal outflow velocities exceed $1000 \mathrm{~km} \mathrm{~s}^{-1}$ and the outflow rates (up to $\sim 1000 M_{\odot} \mathrm{yr}^{-1}$ ) are several times larger than the infrared-based SFRs. (2) Independent, spatially resolved CO-emission observations of Mrk 231 with the IRAM/PdB mm-wave interferometer (Feruglio et al. 2010;

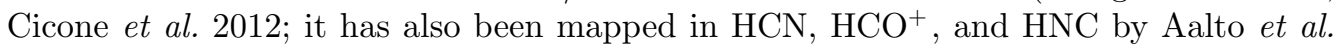
2012) have confirmed this outflow and deduced mass outflow rates of $\sim 700 \mathrm{M}_{\odot} \mathrm{yr}^{-1}$, far larger than the on-going infrared-based $\mathrm{SFR}\left(\sim 160 \mathrm{M}_{\odot} \mathrm{yr}^{-1}\right)$ in the host galaxy.

$\dagger$ Herschel is an ESA space observatory with science instruments provided by European-led Principal Investigator consortia and with important participation from NASA. 
Further mm CO-observations have now been done by our group in a number of the objects discussed here, finding similar CO outflows (Cicone et al. 2014). Remarkably, the CO outflow in Mrk 231 partially overlaps spatially and kinematically with blueshifted optical Na I D 5890, $5896 \AA$ absorption features detected out to $\sim 2-3 \mathrm{kpc}$ from the nucleus (Rupke, Veilleux, \& Sanders 2005c). (3) Our high-resolution Gemini/IFU observations have revealed that the $\mathrm{Na}$ I D outflow in Mrk 231 is wide-angle, thus driven by a QSO wind rather than a jet (Rupke \& Veilleux 2011, hereafter RV11). Similar wide-angle high-velocity outflows have now been mapped at high resolution in a number of other local ULIRGs (e.g., Rupke \& Veilleux 2013a (RV13a) and Rupke \& Veilleux 2013b).

These powerful outflows may be the long-sought "smoking gun" of quasar mechanical feedback that clears out the molecular disk formed from dissipative collapse during the merger. The tentative trend of increasing $\mathrm{OH}$ terminal outflow velocity with increasing AGN luminosity identified by S11 adds support to this idea, but it is based on only 6 ULIRGs (and NGC 253, a regular starburst galaxy). To properly test this idea, one needs to examine a statistically representative sample of ULIRGs and study the properties of their molecular outflows uniformly and systematically. In Veilleux et al. 2013b (hereafter $\mathrm{V} 13 \mathrm{~b}$ ), we report the first results from such a study, focusing on the directly measured outflow velocities. The sample consists of 43 objects, including 38 QUEST ULIRGs and 5 QUEST QSOs. All of these objects show signs of on-going or recent interactions. See V13b for a detailed discussion of the properties of the sample galaxies.

\section{Results}

The main results from this survey (V13b) are:

- The $\mathrm{OH} 119 \mu \mathrm{m}$ feature was detected in 37 (86\%) of the 43 sample galaxies.

- The nature of the $\mathrm{OH} 119 \mu \mathrm{m}$ feature (emission, absorption, or both) depends on the properties of the galaxies: $\mathrm{OH}$ emission is stronger relative to $\mathrm{OH}$ absorption in quasardominated systems, becoming completely dominant (i.e. pure $\mathrm{OH}$ emission) in objects where the quasar contributes more than $\sim 90 \%$ to the bolometric luminosity (called the AGN fraction for short). This behavior is similar to that of the silicate $9.7 \mu \mathrm{m}$ feature studied with Spitzer (Veilleux et al. 2009b) and the H I 21-cm feature measured with GBT in a subset of ULIRGs and QSOs (Teng et al. 2013).

- Unambiguous evidence for molecular outflows, based on the presence of absorption features with median velocities more blueshifted than $-50 \mathrm{~km} \mathrm{~s}^{-1}$, is seen in $26(70 \%)$ of the 37 targets with detected $\mathrm{OH} 119 \mu \mathrm{m}$. Given possible projection effects, this wind detection rate is a lower limit and is consistent with all ULIRGs having molecular winds with an average opening angle $\sim 145^{\circ}$. In contrast, absorption features with median velocities above $+50 \mathrm{~km} \mathrm{~s}^{-1}$, indicative of inflow, are detected in only four objects, none of which show clear inverted P Cygni profiles. This result suggests that molecular inflows subtend a relatively small fraction of $4 \pi$ steradians (e.g., planar or filamentary geometry).

- Typical median outflow velocities are $\sim-200 \mathrm{~km} \mathrm{~s}^{-1}$, but terminal outflow velocities of $\sim-1000 \mathrm{~km} \mathrm{~s}^{-1}$ are detected in several objects. Deprojected outflow velocities will be even larger. The kinematics of these molecular outflows do not show any obvious dependence on the properties of the host galaxies (e.g., near-infrared derived stellar velocity dispersions, stellar masses) and star formation rates, although our sample covers a range of properties that is narrower than that of low- $z$ non-molecular wind studies, where trends have been observed with these quantities.

- There is a tendency for objects with dominant and luminous AGN to show more blueshifted $\mathrm{OH}$ velocities (faster outflows; Figs. 1-2). Our data favor a steepening of the 

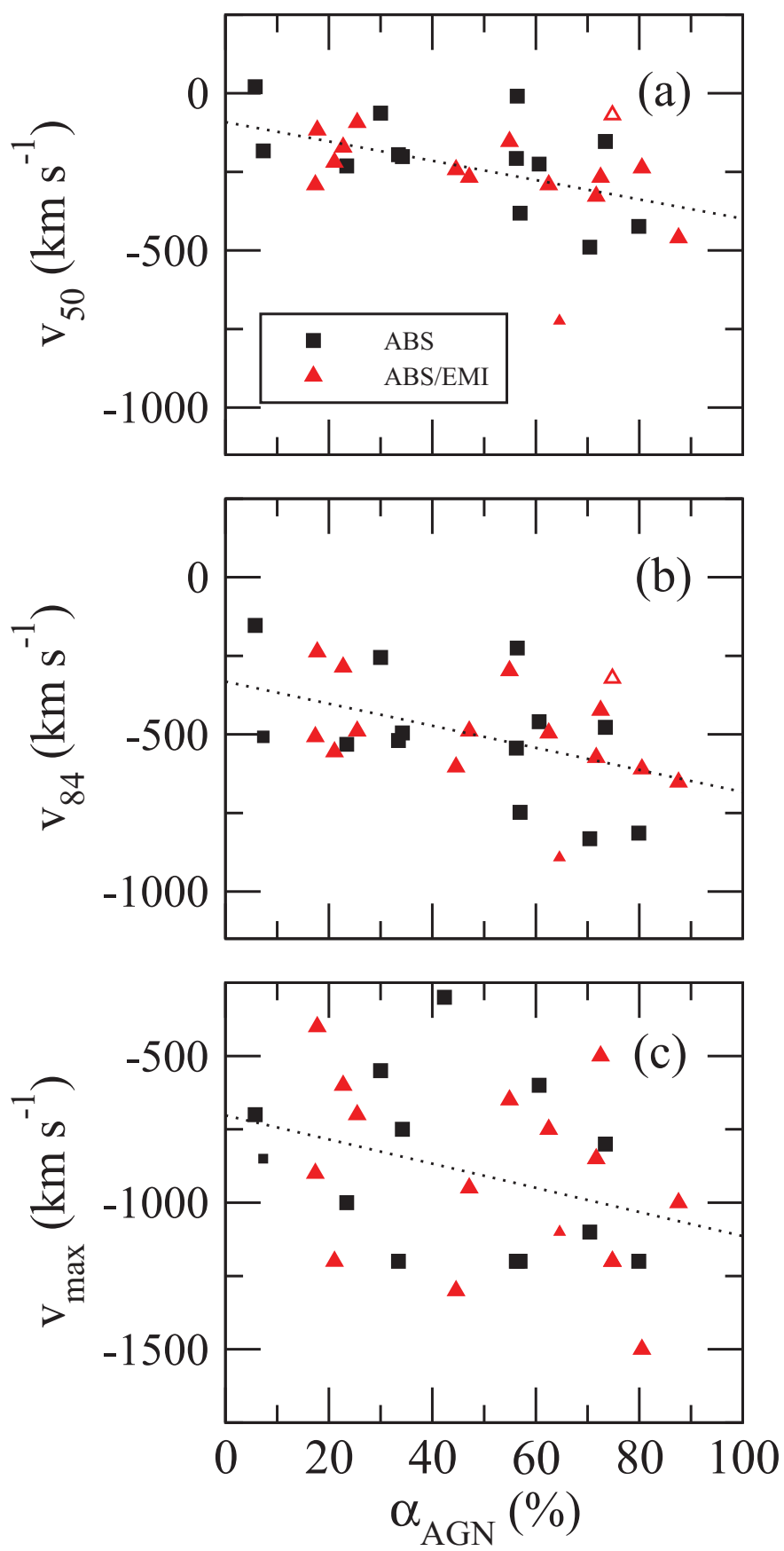

Figure 1. The 50\%, 84\%, and terminal OH outflow velocities as a function of the AGN fractions. The meanings of the symbols are as follows: Filled black squares and filled red triangles represent ULIRGs with $\mathrm{OH} 119 \mu \mathrm{m}$ seen purely in absorption and composite absorption/emission, respectively. The four objects with significant inflows $\left(50 \% \mathrm{OH}\right.$ velocities above $\left.50 \mathrm{~km} \mathrm{~s}^{-1}\right)$ are not shown here. Dotted lines indicate the best linear fits through the data. A significant linear correlation is present with $v_{50}$ and $v_{84}(\mathrm{P}[$ null $] \leqslant 0.05)$, but only tentatively with $v_{\max }(\mathrm{P}[$ null $]$ $\sim 0.07$ ). F12072-0444, indicated by an open red triangle, was not included in the evaluations of these correlations because it is not clear which of the two nuclei is responsible for the $\mathrm{OH}$ absorption feature (both nuclei are included in the PACS entrance aperture). Typical uncertainties on $v_{50}$ and $v_{84}$ are $\pm 50 \mathrm{~km} \mathrm{~s}^{-1}$ and $\pm 200 \mathrm{~km} \mathrm{~s}^{-1}$ on $v_{\max }$. The smaller symbols have larger uncertainties. 

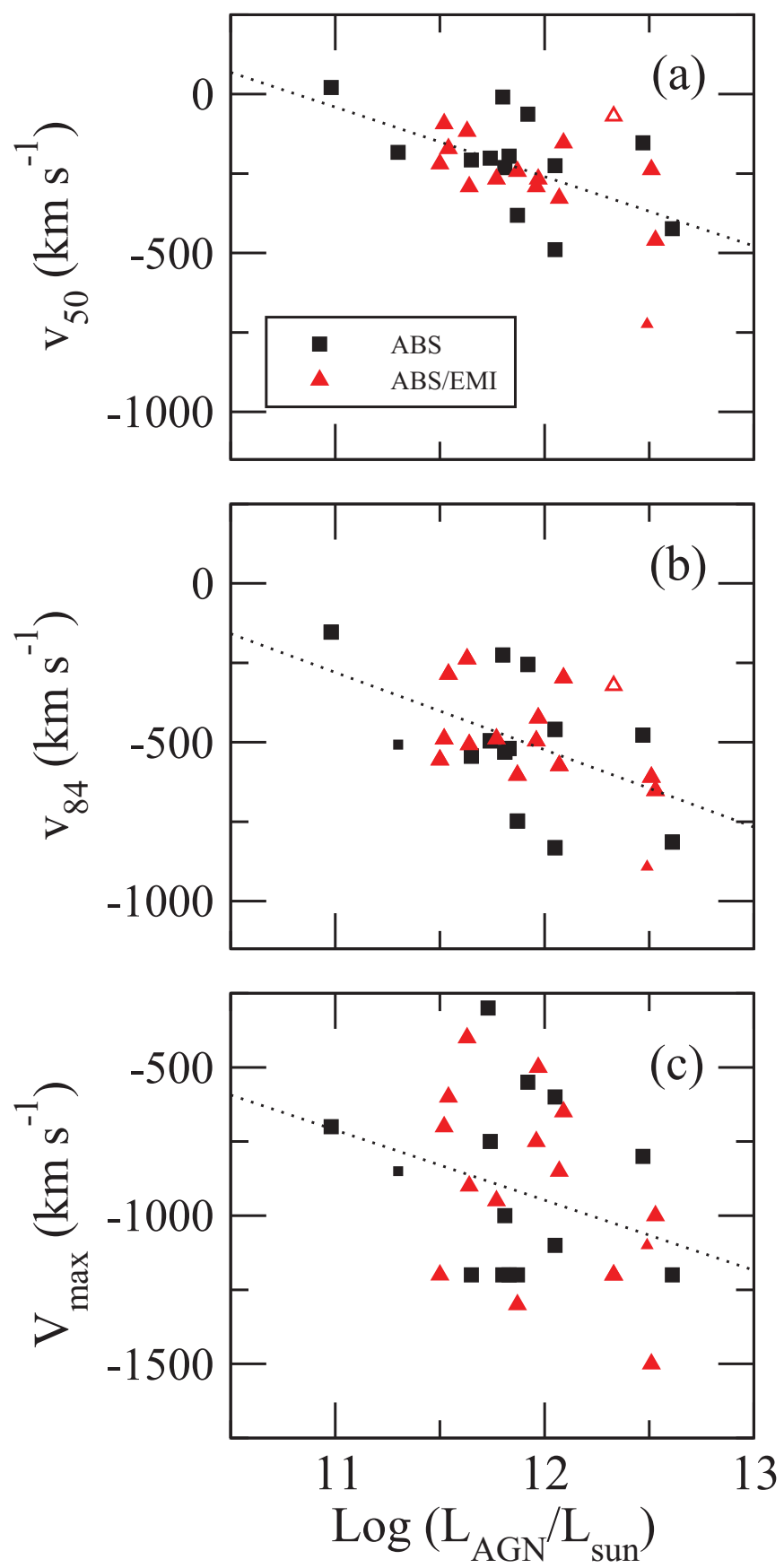

Figure 2. The $50 \%, 84 \%$, and terminal $\mathrm{OH}$ outflow velocities as a function of the AGN luminosities. The meanings of the symbols are the same as in Figure 1. The four objects with significant inflows $\left(50 \% \mathrm{OH}\right.$ velocities above $\left.50 \mathrm{~km} \mathrm{~s}^{-1}\right)$ are not shown here. Dotted lines indicate the best linear fits through the data. A significant linear correlation is present with $v_{50}$ and $v_{84}(\mathrm{P}[$ null $] \leqslant 0.05)$, but only tentatively with $v_{\max }(\mathrm{P}[$ null $] \sim 0.09)$. F12072-0444, indicated by an open red triangle, was not included in the evaluations of these correlations because it is not clear which of the two nuclei is responsible for the $\mathrm{OH}$ absorption feature (both nuclei are included in the PACS entrance aperture). Typical uncertainties on $v_{50}$ and $v_{84}$ are $\pm 50 \mathrm{~km} \mathrm{~s}^{-1}$ and $\pm 200 \mathrm{~km} \mathrm{~s}^{-1}$ on $v_{\max }$. The smaller symbols have larger uncertainties. 
relation between outflow velocities and the logarithms of the AGN luminosities above $\log \left(L_{\mathrm{AGN}}^{\text {break }} / L_{\odot}\right)=11.8 \pm 0.3$, although better statistics are needed, particularly at lower starburst and AGN luminosities, to confirm this non-linearity. While the trend between outflow velocities and AGN fractions may be explained as an obscuration effect, where both the AGN and central high-velocity outflowing material are more easily detectable when the dusty material has been swept away or is seen more nearly face-on, the dependence of the velocities on the AGN luminosities suggests that the AGN is playing a role in driving the fast winds. Our results emphasize that the use of the terminal velocities as the sole measures of outflow velocities should be avoided due to the much larger uncertainties on these measurements.

- The $\mathrm{OH}$ emission profiles in three of the four late-stage ULIRG/QSO mergers with AGN fractions above $\sim 90 \%$ are narrow, despite their large AGN luminosities, suggesting that the winds in these objects, if present, often have modest velocities. Quasar feedback thus seems to subside among mergers that have cleared a path through the dusty circumnuclear material.

\section{Implications}

The results from S11 on 6 ULIRGs in common with our sample indicate that the $\mathrm{OH}$ outflows are not only fast but also powerful, capable of displacing a significant fraction of the entire molecular ISM of the host galaxies. Given our methods of selection, there is no reason to believe that the objects studied here are any different from those in S11. Since the molecular ISM is the fuel for future star formation and galaxy build-up, the quasar-driven outflows detected here may therefore have a significant effect on the evolution of their hosts, potentially quenching star formation in their centers. The modest $\mathrm{OH}$ emission line widths in the infrared-faint quasars perhaps indicate that AGN feedback subsides once the quasar has poked a substantial hole through its natal "cocoon", although this is based on only four objects.

There has been considerable theoretical support in recent years for the idea of quenching star formation in galaxies via AGN-driven winds (e.g., Narayanan et al. 2008; Hopkins et 2009 and references therein), but relatively little direct observational evidence for it, until recently (e.g., Feruglio et al. 2010; S11; RV11; RV13a). This last paper also points out the possibility of increasing outflow velocities at higher AGN luminosities. Using detailed IFU data on a sample of 6 ULIRGs, all from the QUEST sample, RV13a find that fast AGN-driven neutral (Na I D) and/or ionized $(\mathrm{H} \alpha)$ winds are only present in systems with $\log \left(L_{\mathrm{AGN}}^{\mathrm{break}} / L_{\odot}\right) \geqslant 11.7$. This AGN luminosity, while only tentatively identified in that paper since it is based on a very small sample, is consistent to within the uncertainties to that inferred from the larger set of Herschel data. This suggests that the conditions to drive fast neutral/ionized outflows are approximately the same as for the molecular outflows.

In this context, it is instructive to compare the $\mathrm{OH}$ velocities measured here with the Na I velocities measured by Rupke, Veilleux, \& Sanders (2005a), Rupke, Veilleux, \& Sanders (2005b), Rupke, Veilleux, \& Sanders (2005c), and Krug et al. (2014, in prep.) from integrated spectra and RV13a from IFU data (Figure 13 of V13b). Also shown in this figure for comparison are the mid-infrared fine-structure line outflow velocities deduced by Spoon \& Holt (2009) from Spitzer integrated spectra, the $\mathrm{H} \alpha$ velocities measured by RV13a from the IFU data, and the H I 21-cm velocities measured by Teng, Veilleux, \& Baker (2013) from GBT integrated spectra. While the various velocities are measured over different scale ( $\leqslant 200 \mathrm{pc}$ for the $\mathrm{OH}$ velocities according to $\mathrm{S} 11$ and $\leqslant 1-2 \mathrm{kpc}$ for the neutral and ionized gas velocities), general trends are observed: The outflow velocities of 
the molecular gas are often comparable to the velocities of the neutral gas, but they are generally smaller (more positive) than the velocities of the ionized gas (panels $b$ and $c$ in Figure 13 of V13b). The similarity between the $\mathrm{OH}$ and $\mathrm{Na}$ I velocities is particularly evident in panel (b) of Figure 13 of V13b where the sometimes very large nuclear $\mathrm{Na} I$ and $\mathrm{H} \alpha$ outflow velocities, produced on sub-pc scale (e.g., Mrk 231; Veilleux et al. 2013a; Figure 13a of V13b), are excluded from the means. This panel also reiterates the result found by RV13a that the ionized gas is often, but not always, the fastest component of the outflow. While dust obscuration may explain some of these variations (especially for the highly ionized gas found near the center of AGN-dominated ULIRGs; e.g., blue wings in [Ne V] emission profiles of Spoon \& Holt 2009), it likely does not explain all of the spatial variations found in the IFU data.

These multi-phase comparisons may help constrain the processes involved in accelerating the material entrained in the wind. In most cases, the acceleration $a=F / m$ is expected to depend strongly on the ISM phase. For instance, in the case of ram pressure acceleration of clouds in a fast wind or a jet (e.g., Strel'nitskii \& Sunyaev 1973; Chevalier \& Clegg 1985), the force on a spherical cloud of mass $M_{\text {cloud }}$ and density $\rho_{\text {cloud }}$ is expected to scale as the area of the cloud or $\left(M_{\text {cloud }} / \rho_{\text {cloud }}\right)^{2 / 3}$ and thus we expect the acceleration to scale as $\left(M_{\text {cloud }} \rho_{\text {cloud }}^{2}\right)^{-1 / 3}$ (we neglect gravity). Naively, one would expect the molecular clouds to be considerably denser and more massive, on average, than the ionized gas clouds so one would expect the molecular material to always move at lower velocities. This simple-minded argument may be relaxed if each accelerated cloud has a multi-phase structure (e.g., ionized skin or halo surrounding a neutral/molecular core) and thus contributes all at once to the molecular, neutral, and ionized phases of the outflow. But the significant difference in velocities often seen between the ionized and molecular gas phases (Figure 13 of V13b) indicates that this situation cannot apply to the bulk of the outflowing material since the difference in velocities between the various phases would imply severe shearing/shredding and very short cloud lifetimes $\left[\sim R_{\text {cloud }} / \Delta v \sim 10^{4}\left(R_{\text {cloud }} / \mathrm{pc}\right)\left(100 \mathrm{~km} \mathrm{~s}^{-1} / \Delta v\right)\right.$ years, where $R_{\text {cloud }}$ is the cloud radius and $\Delta v$ is the difference in velocities between the various gas phases].

Another possible source of acceleration for these winds is UV-IR radiation pressure. It has the distinct characteristic to be less efficient at accelerating ionized material than dusty neutral/molecular material due to the different sources of opacity. Within the dust sublimation radius, $R_{\text {subl }} \sim L_{46}^{1 / 2}$ pc (where a dust sublication temperature $T_{\text {subl }} \simeq 1200$ $\mathrm{K}$ was assumed), the dominant source of opacity is electron scattering and the corresponding luminosity condition to drive a (spherical) wind is the well-known Eddington luminosity. Estimates of the black hole masses $M_{\mathrm{BH}}$ in our sample galaxies range from $\sim 0.5-5 \times 10^{8} M_{\odot}$ (e.g, Vestergaard \& Peterson 2006; Veilleux, Kim, \& Sanders 2002; Veilleux et al. 2009a), corresponding to Eddington luminosities $L_{\mathrm{Edd}}=1.3 \times 10^{46}$ $\left(M_{B H} / 10^{8}\right)=0.65-6.5 \times 10^{46} \mathrm{ergs} \mathrm{s}^{-1}$. Systems near the AGN luminosity break identified in our data are therefore accreting at sub-Eddington values $\Gamma \equiv L_{\mathrm{AGN}}^{\mathrm{break}} / L_{\mathrm{Edd}} \sim$ $0.04-0.4$.

Outside the dust sublimation radius, the much larger dust opacity prevails and UV-IR radiation pressure from the AGN may drive significant winds. In this situation where dust opacity dominates, Murray, Quataert, \& Thompson (2005) have derived a handy expression for the limiting, Eddington-like luminosity above which momentum deposition from the quasar (and/or starburst) is presumed to be enough to clear most of the gas out of galactic nuclei (i.e. this is a criterion to "blow away" the gas rather than just a "blow out" in the nomenclature of De Young \& Heckman 1994):

$$
\left.L_{M} \simeq\left(4 f_{g} c\right) / G\right) \sigma^{4} \simeq 3 \times 10^{46}\left(f_{g} / 0.1\right)\left(\sigma / 200 \mathrm{~km} \mathrm{~s}^{-1}\right)^{4} \mathrm{ergs} \mathrm{s}^{-1},
$$


where $f_{g}$ is the gas fraction of the host and $\sigma$ is the stellar velocity dispersion, a measure of the depth of the gravitational potential well. In our sample galaxies, $f_{g} \simeq 0.1$ on average, although there is considerable scatter among ULIRGs (e.g., Downes \& Solomon 1998; Combes et al. 2013 and references therein), while $\sigma$ ranges from $\sim 120$ to $\sim 280$ $\mathrm{km} \mathrm{s}^{-1}$ with an average value of $\sim 200 \mathrm{~km} \mathrm{~s}^{-1}$ (as mentioned in $\S 4.4$ of V13b, this value can be considered a lower limit, Rothberg \& Fischer 2010; Rothberg et al. 2013). The steep dependence of $L_{M}$ on $\sigma$ implies that $L_{\mathrm{AGN}}^{\text {break }} \simeq 2-100 \%$ of $L_{M}$, with an average value of $\sim 10 \%$, i.e. the AGN luminosity break above which the AGN plays a dominant role in driving the outflowing molecular gas in local ULIRGs is generally lower than the Eddington-like luminosity derived by Murray et al. (2005). The fact that $L_{\mathrm{AGN}}^{\mathrm{break}}$ is lower than $L_{M}$ is not surprising since the latter quantity is the maximum luminosity an object may have before blowing away all of the gas from a galaxy via radiation pressure on dust. For more details, see V13b.

\section{Acknowledgements}

Support for this work was provided by NASA through Herschel contracts 1427277 and 1454738 (S. V. and M. M.) and contracts 1364043, 1435724, and 1456609 (J. F.).

\section{References}

Aalto, S., et al. 2012, A\& A, 537, 44

Chevalier, R. A. \& Clegg, A. W. 1985, Nature, 317, 44

Cicone, C., et al. 2012, A\& $A, 543,99$

Cicone, C., et al. 2014, A\&A, 000, 000 (arXiv:1311.2595)

Combes, F., et al. 2013, A\&A, 550, 41

De Young, D. \& Heckman, T. M. 1994, ApJ, 431, 598

Downes, D. \& Solomon, P. M. 1998, ApJ, 507, 615

Feruglio, C., et al. 2010, A\& $A$, 518, L155

Fischer, J., Sturm, E., González-Alfonso, E., et al. 2010, A\&A, 518, L41 (F10)

González-Alfonso, E., et al. 2012, A\&A, 541, 4

González-Alfonso, E., et al. 2014, A\&A, 561, 27

Hopkins, P. F., et al. 2009, MNRAS, 398, 303

Murray, N., Quataert, E., \& Thompson, T. A. 2005, ApJ, 618, 569

Narayanan, D., et al. 2008, ApJS, 176, 331

Rothberg \& Fischer 2010, ApJ, 712, 318

Rothberg, B., et al. 2013, ApJ, 767, 72

Rupke, D. S. N. \& Veilleux, S. 2011, ApJ, 729, L27 (RV11)

Rupke, D. S. N. \& Veilleux, S. 2013a, ApJ, 768, 75 (RV13a)

Rupke, D. S. N. \& Veilleux, S. 2013b, ApJ, 775, L15

Rupke, D. S., Veilleux, S., \& Sanders, D. B. 2005a, ApJS, 160, 87

Rupke, D. S., Veilleux, S., \& Sanders, D. B. 2005b, ApJS, 160, 115

Rupke, D. S., Veilleux, S., \& Sanders, D. B. 2005c, ApJ, 632, 751

Spoon, H. \& Holt, J. 2009, ApJ, 702, L42

Strel'nitskii, V. S. \& Sunyaev, R. A. 1973, Soviet Astronomy, 16, 579

Sturm, E., et al. 2011, ApJ, 733, L16 (S11)

Teng, S. H., Veilleux, S., \& Baker, A. J. 2013, ApJ, 765, 95

Veilleux, S., Kim, D.-C., \& Sanders, D. B. 2002, ApJS, 143, 315

Veilleux, S., Kim, D.-C., et al. 2009a, ApJ, 701, 587

Veilleux, S., Rupke, D. S. N., et al. 2009b, ApJS, 182, 628

Veilleux, S., Trippe, M., et al. 2013a, ApJ, 764, 15

Veilleux, S., Meléndez, M., et al. 2013b, ApJ, 776, 27 (V13b)

Vestergaard, M. \& Peterson, B. M. 2006, ApJ, 641, 689 